\title{
Jaw and Long Bone Marrows Have a Different Osteoclastogenic Potential
}

\author{
Ana Paula de Souza Faloni - Ton Schoenmaker • \\ Azin Azari • Eduardo Katchburian • \\ Paulo S. Cerri · Teun J. de Vries • Vincent Everts
}

Received: 27 May 2010/Accepted: 28 August 2010/Published online: 23 September 2010

(C) The Author(s) 2010. This article is published with open access at Springerlink.com

\begin{abstract}
Osteoclasts, the multinucleated bone-resorbing cells, arise through fusion of precursors from the myeloid lineage. However, not all osteoclasts are alike; osteoclasts at different bone sites appear to differ in numerous respects. We investigated whether bone marrow cells obtained from jaw and long bone differed in their osteoclastogenic potential. Bone marrow cells from murine mandible and tibiae were isolated and cultured for 4 and 6 days on plastic or 6 and 10 days on dentin. Osteoclastogenesis was assessed by counting the number of TRAP ${ }^{+}$multinucleated cells. Bone marrow cell composition was analyzed by FACS. The expression of osteoclast- and osteoclastogenesis-related genes was studied by qPCR. TRAP activity and resorptive activity of osteoclasts were measured by absorbance and morphometric analyses, respectively. At day 4 more osteoclasts were formed in long bone cultures than in jaw cultures. At day 6 the difference in number was no longer observed. The jaw cultures, however, contained more large osteoclasts on plastic and on dentin. Long bone marrow contained more
\end{abstract}

The authors have stated that they have no conflict of interest.

A. P. de Souza Faloni - E. Katchburian

Department of Morphology and Genetics, Federal University of São Paulo (UNIFESP), Rua Botucatu, 740 Vila Clementino, São Paulo, SP 04023-900, Brazil

A. P. de Souza Faloni · P. S. Cerri

Department of Morphology, Dental School, Universidade Estadual Paulista (UNESP), Rua Humaitá 1680, Araraquara, SP 14801-903, Brazil

T. Schoenmaker · A. Azari · T. J. de Vries · V. Everts ( $\square)$ Department of Oral Cell Biology and Periodontology, Academic Center of Dentistry Amsterdam (ACTA), Research Institute Move, University of Amsterdam and VU University Amsterdam, Gustav Mahlerlaan 3004, 1081 LA Amsterdam, The Netherlands e-mail: v.everts@acta.nl osteoclast precursors, in particular the myeloid blasts, and qPCR revealed that the RANKL:OPG ratio was higher in long bone cultures. TRAP expression was higher for the long bone cultures on dentin. Although jaw osteoclasts were larger than long bone osteoclasts, no differences were found between their resorptive activities. In conclusion, bone marrow cells from different skeletal locations (jaw and long bone) have different dynamics of osteoclastogenesis. We propose that this is primarily due to differences in the cellular composition of the bone site-specific marrow.

Keywords Jaw $\cdot$ Long bone $\cdot$ Osteoclastogenesis . Osteoclast precursor $\cdot$ Heterogeneity $\cdot$ Marrow

Osteoclasts are the cells responsible for the degradation of mineralized tissues and can be generated from bone marrow precursors [1] of the myeloid lineage [2, 3].

It has long been considered that all osteoclasts are alike, independent of the skeletal site where they exert their activity. However, a variety of data show that osteoclasts at different bone sites appear to differ and the existence of bone site-specific osteoclast heterogeneity has been proposed (reviewed by Everts et al. [4]). The differences found between osteoclasts from distinct bone sites (e.g., calvaria and long bone) are mainly related to the proteins involved in bone degradation [5-8]. It was demonstrated that calvarial osteoclasts use matrix metalloproteinases as well as cysteine proteinases (e.g., cathepsin K) for resorption, whereas long bone osteoclasts use primarily cysteine proteinases [5-7]. Moreover, in comparison to long bone, calvarial osteoclasts are larger and express a higher level of tartrate-resistant acid phosphatase (TRAP) [8, 9], an iron-containing enzyme related to the degradation of bone constituents [10-14] and intracellular vesicular trafficking 
in the osteoclast [15]. In addition to these differences, anion exchanger-2 (AE-2) proved to be essential for resorption by long bone osteoclasts but less important for resorption by calvarial osteoclasts. The latter cells express also the sodium transporter Slc4a4, which seems to compensate for the loss of AE-2 [16].

Although never proven, osteoclast heterogeneity can be seen as an adaptation to different bones. Indeed, calvaria and long bones differ in the mode of ossification and, consequently, in the composition of their matrix, regarding not only the amount of collagen and noncollagenous protein but also the level of collagen cross-linking [17, 18]. The differences in the bone matrix are considered to be related to the distinct proteolytic pathways osteoclasts use to degrade bone [4].

With respect to the question of how different osteoclasts arise at different bone locations, one possibility is the presence of different osteoclast precursors. Mouse bone marrow contains diverse osteoclast precursors, all of which have the propensity to differentiate into osteoclasts $[2,3]$. Among the osteoclast precursors from the myeloid differentiation pathway, myeloid blasts more rapidly differentiate into multinucleated cells, whereas cells earlier (early blasts) and later (monocytes) in differentiation need more time. Already after 4 days of culture with receptor activator of nuclear factor- $\kappa \mathrm{B}$ ligand (RANKL) and macrophage colony-stimulating factor (M-CSF), myeloid blastderived osteoclasts are formed and the expression of osteoclast-related genes is higher in this culture in comparison to those containing early blasts and monocytes. Moreover, earlier and more bone resorption is observed from myeloid blast-derived osteoclasts [3].

Besides the differences mentioned above, osteoclasts from distinct bone sites seem to respond differently to some cytokines. RANKL and osteoprotegerin (OPG) are cytokines related to osteoclast formation, and the RANKL:OPG ratio is frequently used as a measure to indicate whether a system has the potential to generate osteoclasts [19]. When this ratio is favorable to osteoclastogenesis, the RANK-RANKL interaction will lead to differentiation of the osteoclast precursor into osteoclasts [20]. In RANKLdeficient mice, the effect of transgenically induced RANKL expression by $\mathrm{T}$ and $\mathrm{B}$ cells normalizes osteoclast formation in long bones but not in the jaw. Therefore, the failure of RANKL-expressing $\mathrm{T}$ and $\mathrm{B}$ cells on bone resorption in the jaws is site-specific [21].

Recently, the observation that bisphosphonates may cause necrosis of the jaw whereas long bones appear unaffected by this drug [22, 23] may suggest that osteoclasts from the jaw may respond differently to bisphosphonates and that this type of bone may thus harbor its own type of osteoclasts.

In an attempt to shed light on possible differences between osteoclasts generated from jaw in comparison with long bone, lower jaw (jaw) and tibial (long bone) bone marrow were cultured in the presence of RANKL and MCSF on plastic or on dentin. We evaluated the osteoclastogenic potential of these different cultures and analyzed the expression of various osteoclast- and osteoclastogenesis-related genes.

\section{Materials and Methods}

\section{Bone Marrow Isolation}

Permission for the animal experiments was obtained from the Animal Welfare Committee of the VU University (Amsterdam, The Netherlands). Adequate measures were taken to minimize animal pain or discomfort, and the experiments were compliant with the standards defined by the European Communities Council Directive of 24 November 1986 (86/ 609/EEC). Eight-week-old female C57BL/6J mice (Harlan, Horst, The Netherlands) were killed with a peritoneal injection of a lethal dose of sodium pentobarbitone (Euthesate, $0.1 \mathrm{ml}$ sodium pentobarbital per mouse; Sanofi Santé Animale Benelux, Maassluis, The Netherlands). Tibiae were removed, cleaned of soft tissue, and maintained in Petri dishes containing $\alpha$-minimal essential medium devoid of phenol red ( $\alpha$-MEM; GIBCO, Paisley, UK) supplemented with 5\% fetal calf serum (FCS; HyClone, Logan, UT) and 1\% antibiotics $(100 \mathrm{U} / \mathrm{ml}$ penicillin, $100 \mu \mathrm{g} / \mathrm{ml}$ streptomycin, and $250 \mathrm{ng} / \mathrm{ml}$ amphotericin B [antibiotic antimycotic solution; Sigma, St. Louis, MO]). Heparin (170 IE/ml; Leo Pharmaceutical Products, Weesp, The Netherlands) was added to the medium. For isolation of the bone marrow of the jaw, the lower jaws (Fig. 1) were removed and placed in Petri dishes. Subsequently, the soft tissue was scraped off and the incisors were removed. The anterior part of the lower jaws preceding the molar block and the posterior condylar part distally from the molar block were removed, resulting in jaw bone containing the molars. The bone marrow of both tibiae and jaws were isolated by crushing the bones in mortars [3]. Cell suspensions were aspirated through a 21-gauge needle and filtered over a $100-\mu \mathrm{m}$ pore size Cell Strainer filter (Falcon/Becton Dickinson, Franklin Lakes, NJ).

\section{Osteoclastogenesis}

Bone marrow cells were plated in a 96-well cell culture plate (Cellstar, Greiner Bio-one, Monroe, NC) at a density of $1 \times 10^{5}$ cells/well. Cells were seeded on plastic or on 650 $\mu \mathrm{m}$-thick dentin slices and cultured in $150 \mu \mathrm{l}$ culture medium containing $30 \mathrm{ng} / \mathrm{ml}$ recombinant murine $\mathrm{M}-\mathrm{CSF}$ (R\&D Systems, Minneapolis, MN) and $20 \mathrm{ng} / \mathrm{ml}$ recombinant murine RANKL (RANKL-TEC, R\&D Systems). Culture plates were stored in a humidified atmosphere of $5 \% \mathrm{CO}_{2}$ in 


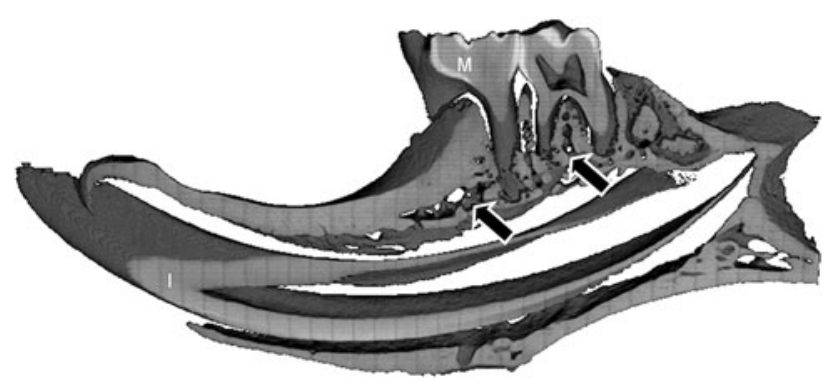

Fig. 1 MicroCT cross section of a mouse lower jaw. Arrows indicate marrow cavities in the molar $(M)$ area. For the isolation of bone marrow of the lower jaw, the incisor $(I)$ and the frontal and distal jaw bone were dissected. The remaining molar block with accompanying bone marrow cavities was crunched, and a cell suspension containing bone marrow was isolated. MicroCT was generously provided by Dr. Lars Mulder (Department of Anatomy, Academic Center for Dentistry Amsterdam, Amsterdam, The Netherlands)

air at $37^{\circ} \mathrm{C}$. Culture medium was collected and replaced after 3 days. At the end of the culture periods, wells were either fixed in 4\% PBS-buffered formaldehyde and stored in PBS at $4^{\circ} \mathrm{C}$ (used for TRAP staining) or washed and stored in water at $4^{\circ} \mathrm{C}$ (for resorption analysis). For quantitative PCR (qPCR) analysis, cells were lysed in RNA lysis buffer from the RNeasy Mini Kit (Qiagen, Hilden, Germany) and stored at $-80^{\circ} \mathrm{C}$ until RNA isolation.

\section{TRAP Staining}

Cells cultured for 4 days on plastic and for 6 days on plastic or on dentin were stained for TRAP activity using the leukocyte acid phosphatase kit (Sigma). Nuclei were stained with diamidino-2-phenylindole dihydrochloride (DAPI). To evaluate osteoclast formation, the number of $\mathrm{TRAP}^{+}$cells with three or more nuclei was counted. These multinucleated cells were categorized in $\mathrm{TRAP}^{+}$cells containing 3-5, 6-10, or more than 10 nuclei in the cultures on plastic and in those on dentin we assessed cells with $3-5,6-10,11-30$, or more than 30 nuclei.

Immunofluorescence Labeling, Flow Cytometry, and Sorting

For immunofluorescence labeling, flow cytometry, and sorting analysis, jaw and long bone marrow cells were submitted to the procedures previously described [3, 24]. Antibodies ER-MP12 and ER-MP20 were a generous gift from Dr. P. Leenen (Department of Immunology, Erasmus University, Rotterdam, The Netherlands). Jaw and long bone marrow cell suspensions were spun down and incubated in biotinylated ER-MP12, recognizing CD31. Subsequently, cells were washed and incubated in 1\% BSA in PBS containing FITC-conjugated ER-MP20, recognizing Ly-6C and streptavidin-PE conjugate (Becton Dickinson, San Jose,
CA). Cells washed and recovered in culture medium were sieved through $50-\mu \mathrm{m}$ filters (Filcons, Becton Dickinson) before cell sorting. Early blasts $\left(\mathrm{CD} 31^{\text {high }} / \mathrm{Ly}-6 \mathrm{C}^{-}\right)$, myeloid blasts $\left(\mathrm{CD} 31^{+} / \mathrm{Ly}-6 \mathrm{C}^{+}\right)$, and monocytes $\left(\mathrm{CD} 31^{-} / \mathrm{Ly}-\right.$ $6 \mathrm{C}^{\text {high }}$ ) were sorted at $3 \times 10^{7}$ cells/hour on FACSAria (Becton Dickinson). Typical profiles are shown in Fig. 3a, b.

\section{RNA Isolation and Real-Time qPCR}

For real-time qPCR analyses, samples were collected at $t=0$ and on days 2,4 , and 6 . RNA from cultured cells was isolated using the RNeasy Mini Kit (Qiagen) according to the manufacturer's instructions. RNA was measured with the NanoDrop (Nanodrop Technologies, Wilmington, MA). RNA (100 ng) was used in the reverse-transcriptase reaction, which was performed according to the cDNA synthesis kit (MBI Fermentas, Vilnius, Lithuania), using both the oligo(dT) 18 and the $\mathrm{D}(\mathrm{N}) 6$ primers. Real-time PCR primers were designed using Primer Express software, version 2.0 (Applied Biosystems, Foster City, CA) (Table 1). To avoid amplification of genomic DNA, each amplicon spanned at least one intron. PCR efficiencies of the different primer sets were comparable.

Real-time PCR was performed on an ABI PRISM 7000 machine (Applied Biosystems). Reactions were performed with $1 \mathrm{ng}$ cDNA in a total volume of $15 \mu \mathrm{l}$ containing SYBR Green PCR Master Mix, consisting of SYBR Green 1 Dye, AmpliTaq Gold DNA polymerase, dNTPs, passive reference, and buffer (Applied Biosystems), and $300 \mathrm{nM}$ of each primer. After an initial activation step of the AmpliTaq Gold DNA polymerase for $10 \mathrm{~min}$ at $94^{\circ} \mathrm{C}, 40$ cycles were run of a two-step PCR consisting of a denaturation step at $95^{\circ} \mathrm{C}$ for $30 \mathrm{~s}$ and annealing and extension at $60^{\circ} \mathrm{C}$ for $1 \mathrm{~min}$. Subsequently, PCR products were subjected to melting curve analysis to test if any unspecific PCR products were generated.

Porphobilinogen deaminase (PBGD) was used as the housekeeping gene. Expression of this gene was not affected by the experimental conditions. Samples were normalized for the expression of PBGD by calculating the $\Delta C_{\mathrm{t}}\left(C_{\text {tgene of interest }}-C_{\mathrm{tPBGD}}\right)$ and expression of the different genes is given as $2^{-(\Delta \mathrm{Ct})}$.

\section{Resorption Assay}

The resorptive activity of osteoclasts generated from precursors of the different bone marrows was evaluated. For this purpose, bone marrow precursors were cultured on dentin. After 10 days of culture, cells were removed from the dentin slices and resorption areas were visualized with Coomassie Brilliant Blue, as previously described [3]. Resorption areas were measured using Image Pro-Plus Software (Media Cybernetics, Silver Spring, MD). 
Table 1 qPCR primers used

\begin{tabular}{|c|c|c|c|}
\hline Primer & Sequence $5^{\prime} \rightarrow 3^{\prime}$ & $\begin{array}{l}\text { Amplicon } \\
\text { length (bp) }\end{array}$ & Ensembl gene ID \\
\hline PBGD & $\begin{array}{l}\text { AgTgATgAAAgATgggCAACt } \\
\text { TCTggACCATCTTCTTgCTgA }\end{array}$ & 122 & ENSMUSG00000032126 \\
\hline NFATc1 & $\begin{array}{l}\text { CATgCgAgCCATCATCgA } \\
\text { TgggATgTgAACTCggAAgAC }\end{array}$ & 129 & ENSMUSG00000033016 \\
\hline DC-STAMP & $\begin{array}{l}\text { TgTATCggCTCATCTCCTCCAT } \\
\text { gACTCCTTgggTTCCTTgCTT }\end{array}$ & 100 & ENSMUSG00000022303 \\
\hline TRAP & $\begin{array}{l}\text { gACAAgAggTTCCAggAgACC } \\
\text { gggCTggggAAgTTCCAg }\end{array}$ & 157 & ENSMUSG00000001348 \\
\hline Cathepsin K & $\begin{array}{l}\text { ACAgCAggATgTgggTgTTCA } \\
\text { gCCgAgAgATTTCATCCACCT }\end{array}$ & 146 & ENSMUSG00000028111 \\
\hline c-Fms & $\begin{array}{l}\text { gAAACgCgACCTTCAAAAACA } \\
\text { ggCCggATCTTTgACATACAA }\end{array}$ & 100 & ENSMUSG00000024621 \\
\hline RANK & $\begin{array}{l}\text { TgggCTTCTTCTCAgATgTCTTT } \\
\text { TgCAgTTggTCCAAggTTTg }\end{array}$ & 59 & ENSMUSG00000026321 \\
\hline RANKL & $\begin{array}{l}\text { CTgAggCCCAgCCATTTg } \\
\text { ggAACCCgATgggATgCT }\end{array}$ & 58 & ENSMUSG00000022015 \\
\hline OPG & $\begin{array}{l}\text { TCCggCgTggTgCAA } \\
\text { ATACAgggTgCTTTCgATgAAgTC }\end{array}$ & 100 & ENSMUSG00000063727 \\
\hline
\end{tabular}

For each gene, the first oligonucleotide sequence represents the forward primer and the second sequence, the reverse primer

higher $(P<0.01)$ than the number formed by the jaw precursors (Fig. 2e). In the jaw cultures, at that time point, most of the $\mathrm{TRAP}^{+}$cells were mononuclear and only few osteoclast-like cells could be observed (Fig. 2a), whereas in the long bone cultures several osteoclasts were already formed (Fig. 2b). When categorized according to number of nuclei, the number of long bone $\mathrm{TRAP}^{+}$multinucleated cells with 3-5 nuclei and with 6-10 nuclei was significantly higher $(P<0.01)$ than in the jaw cultures. The larger osteoclasts containing more than 10 nuclei were present on day 4 in the long bone cultures but hardly found in the jaw cultures $(P<0.05)$ (Fig. 2f).

After 6 days of culture, the total number of osteoclasts formed by jaw and long bone precursors was no longer different (Fig. 2c-e). Compared to 4 days, approximately three times more $(P<0.05)$ osteoclasts were formed by jaw bone marrow at day 6 , whereas no increase was observed in long bone marrow. When categorized in osteoclasts with 3-5 or 6-10 nuclei, no differences were observed between long bone and jaw osteoclasts. However, the number of large osteoclasts with more than 10 nuclei was significantly higher $(P<0.01)$ in jaw cultures (Fig. 2f). Although large osteoclasts were observed at both time points (days 4 and 6) and in both cultures, most of the $\mathrm{TRAP}^{+}$cells contained 3-5 nuclei.

Long Bone Marrow Contains Higher Percentages of Myeloid Blasts and Early Blasts

Based on the CD31 and Ly-6C antigen expression, the two bone marrow fractions were analyzed and the percentages 

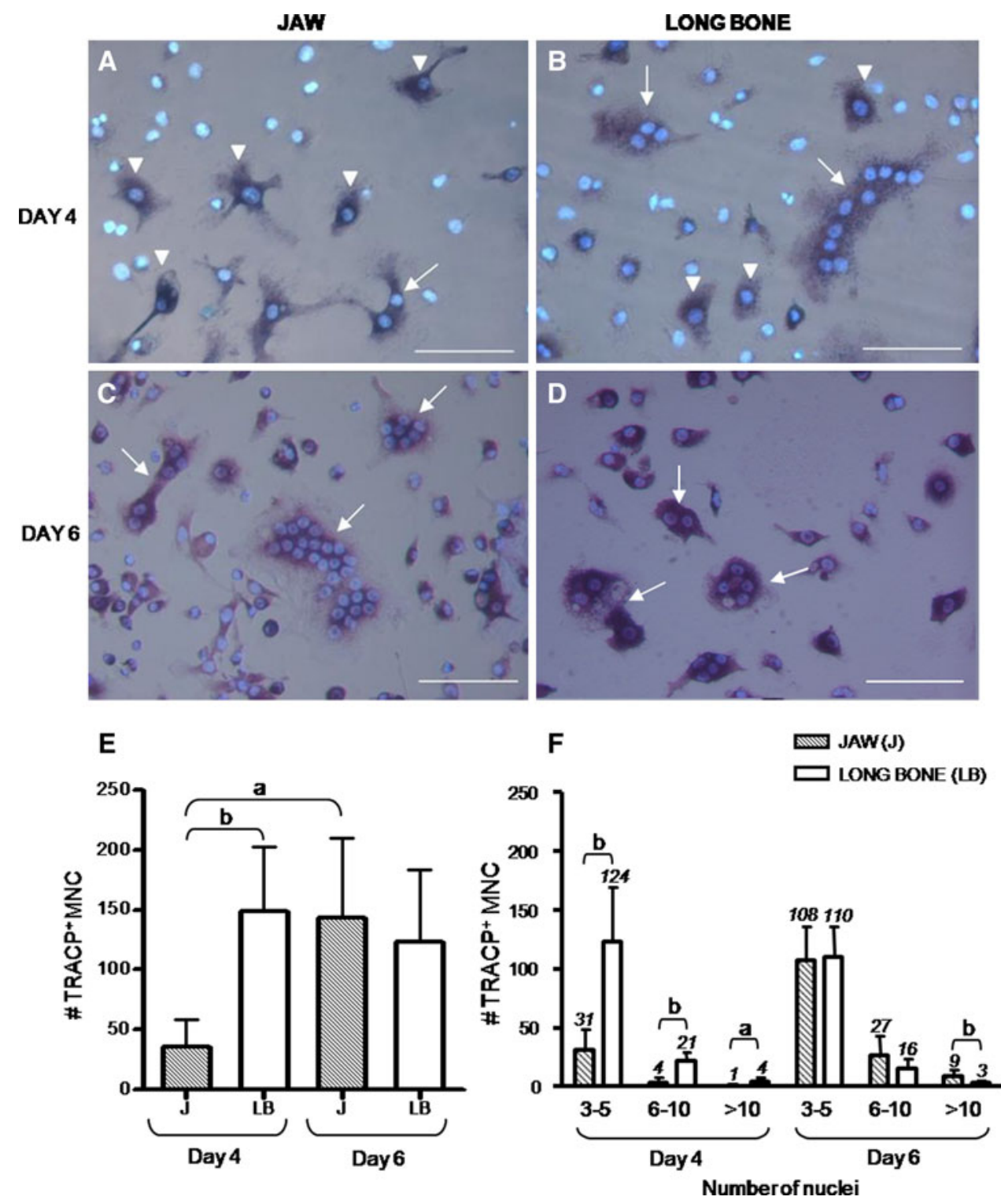

Fig. 2 TRAP $^{+}$multinucleated cells formed from jaw (a, c) and long bone $(\mathbf{b}, \mathbf{d})$ precursors, after $4(\mathbf{a}, \mathbf{b})$ and $6(\mathbf{c}, \mathbf{d})$ days of culture on plastic. a Most of the TRAP $^{+}$cells shown here are mononuclear (arrowheads) and only one cell has more than one nucleus (arrow). b Large $\mathrm{TRAP}^{+}$multinucleated cells (arrows) are apparent, and only a few TRAP $^{+}$mononucleated cells are observed (arrowheads). c Large $\mathrm{TRAP}^{+}$multinucleated cells with several nuclei (arrows) are seen. d Small TRAP $^{+}$multinucleated cells (arrows) are observed. TRAP reaction counterstained with DAPI. Bars $=100 \mu \mathrm{m}$. e Total number of $\mathrm{TRAP}^{+}$multinucleated cells formed by the jaw and long bone precursors, after 4 and 6 days of culture on plastic. On day 4, the number of $\mathrm{TRAP}^{+}$multinucleated cells formed by the long bone

precursors is approximately threefold higher than in the jaw cultures. This difference is no longer apparent at day 6 . f If the TRAP ${ }^{+}$ multinucleated cells are categorized according to the number of nuclei, on day 4 , the number of osteoclasts exhibiting $3-5,6-10$, and more than 10 nuclei is significantly higher in the long bone cultures in comparison to the jaw cultures. On day 6 , this difference is no longer observed. However, on day 6, the number of large osteoclasts with more than 10 nuclei is significantly higher in the jaw cultures. For both periods (4 and 6 days), most of the osteoclasts contained 3-5 nuclei. Data are expressed as mean \pm SD. The mean number of cells is given on top of each bar. Results from four experiments $(n=3$ mice per experiment) are shown. ${ }^{\mathrm{a}} P<0.05,{ }^{\mathrm{b}} P<0.01$

of early blasts $\left(\mathrm{CD} 31^{\text {high }} / \mathrm{Ly}-6 \mathrm{C}^{-}\right)$, myeloid blasts $\left(\mathrm{CD} 31^{+} /\right.$ Ly- $\left.6 \mathrm{C}^{+}\right)$, and monocytes $\left(\mathrm{CD} 31^{-} / \mathrm{Ly}-6 \mathrm{C}^{\text {high }}\right)$ were assessed (Fig. 3a, b). Among the total fractions of bone marrow cells, long bone contained significantly higher percentages of cells from the myeloid lineage-early blasts $(P<0.001)$, myeloid blasts $(P<0.001)$, and monocytes
$(P<0.01)$-in comparison with the jaw (Fig. 3c). Among the cells from the myeloid lineage which could give rise to osteoclasts, the percentages of myeloid blasts $(P<0.01)$ and early blasts $(P<0.05)$ were higher in the long bone marrow, whereas the percentage of monocytes $(P<0.01)$ was higher in the jaw bone marrow (Fig. 3d). 
A
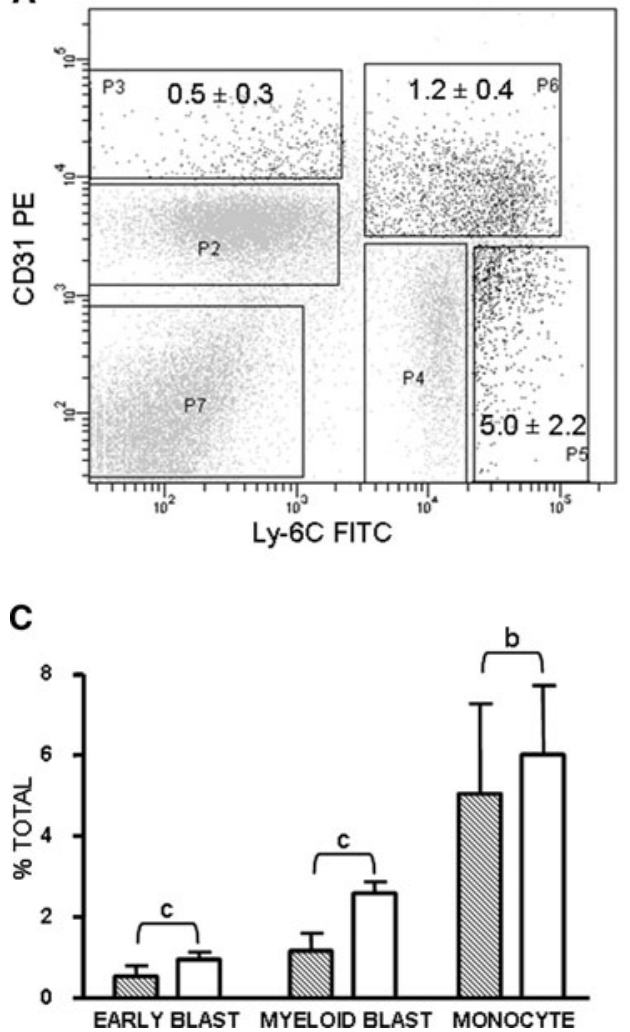

Fig. 3 Two-color flow-cytometric analysis of mouse bone marrow from jaw (a) and long bone (b). Cells were labeled with anti-CD31 and anti-Ly-6C. Percentages of cells found per gated area are indicated ( $n=12$ mice from four experiments). Bold lettering: subpopulations highly enriched in early blasts $(P 3)$, myeloid blasts $(P 6)$, and monocytes (P5). Other fractions mainly contain lymphocytes (P2), erythroid blasts (P7), or granulocytes (P4). c Among the total population of bone marrow cells, the percentage of myeloid cells

Distinct Expression Pattern of Osteoclastand Osteoclastogenesis-Related Genes by Jaw and Long Bone Cells Over Time

We analyzed the expression over time of the osteoclastrelated genes NFATc1 (nuclear factor of activated T cells c1), TRAP, cathepsin K, and DC-STAMP (dendritic cellspecific transmembrane protein) (Fig. 4a-d) and the osteoclastogenesis genes c-FMS (M-CSF receptor), RANK, RANKL, and OPG (Fig. 4e-h) in jaw and long bone marrow cultures, upon stimulation with M-CSF and RANKL.

At $t=0$, before culture, when the precursors were collected from the two marrows, gene expression was negligible for all genes analyzed. From $t=0$ all genes were upregulated. Until day 2, the expression of the genes was similar between the two types of cultures (Fig. $4 a-f$ ). However, from day 2 to day 4, gene expression of both osteoclast- and osteoclastogenesis-related genes had a steeper increase in long bone cultures.
B
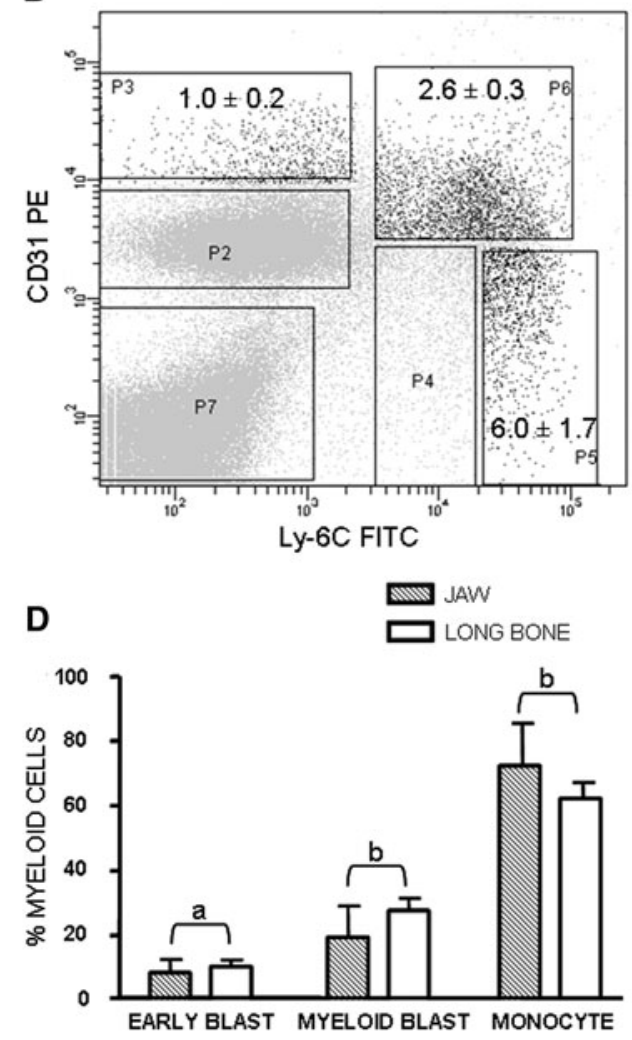

(early blasts, myeloid blasts, and monocytes) was significantly higher in the long bone marrow in comparison with the jaw. d Within the myeloid cells, the number of myeloid blasts and early blasts were higher in long bone marrow, whereas jaw bone marrow contained relatively more monocytes. The other fractions mainly contained lymphocytes, erythroid blasts, or granulocytes. Data are expressed as mean $\pm \mathrm{SD} .{ }^{\mathrm{a}} P<0.05,{ }^{\mathrm{b}} P<0.01,{ }^{\mathrm{c}} P<0.001$

Typically, expression increased until day 4 for long bone precursors, followed by a drop in expression. Expression by precursors isolated from the jaw, however, increased up to day 6 when they reached the same level of expression as long bone precursors.

To gain insight into the increase in gene expression between days 2 and 4, we calculated the t4:t2 ratio. From day 2 to day 4, there was a higher increase in the expression of most of the genes from the long bone precursors in comparison with the jaw. Statistical analyses of the $t 4: t 2$ ratio showed that in long bone cultures, from day 2 to day 4 , there was a higher increase in the expression of c-FMS $(P<0.01)$, RANKL $(P<0.05)$, OPG $(P<0.01)$, TRAP $(P<0.05)$, and cathepsin $\mathrm{K}(P<0.01)$ in comparison with the jaw cultures. Another difference between jaw and long bone was the distinct gene-expression patterns of day 4 and day 6 . In this period, gene expression by the long bone cells decreased (Fig. 4a-f).

Expression patterns of RANKL and OPG (Fig. $4 \mathrm{~g}, \mathrm{~h}$ ) were distinct compared to the other genes. RANKL was 
Fig. 4 Kinetics of gene expression in bone marrow cultures from jaw and long bone stimulated with RANKL and MCSF. Expression was assessed at $t=0$ and on days 2, 4, and 6 . During this culture period, the genes NFATc-1 (a), TRAP (b), cathepsin K (c), DC-STAMP (d), c-FMS (e), and RANK (f) were upregulated. For long bone cultures, highest expression was reached on day 4 for most of the genes. From day 2 to day 4 , long bone cultures tended to show a higher increase in gene expression than jaw cells. From day 4 to day 6 , most of the genes in the long bone cultures showed a decrease in expression, whereas the jaw gene expression continued to increase or remained at the same level. Expression of RANKL (g) and OPG (h) was distinct from that of the other genes. On day 2, expression of RANKL was significantly higher in long bone cultures in comparison to jaw cultures. Overall, except for $t=0$ (when long bone

expression was higher than that from the jaw), expression of OPG was significantly higher for precursors and cultures from jaw. Expression was normalized for PBGD, expression of which was stable for all samples.

Results (mean \pm SEM)

obtained from two experiments ( $n=3$ mice per experiment) are shown. ${ }^{\mathrm{a}} P<0.05,{ }^{\mathrm{b}} P<0.01$
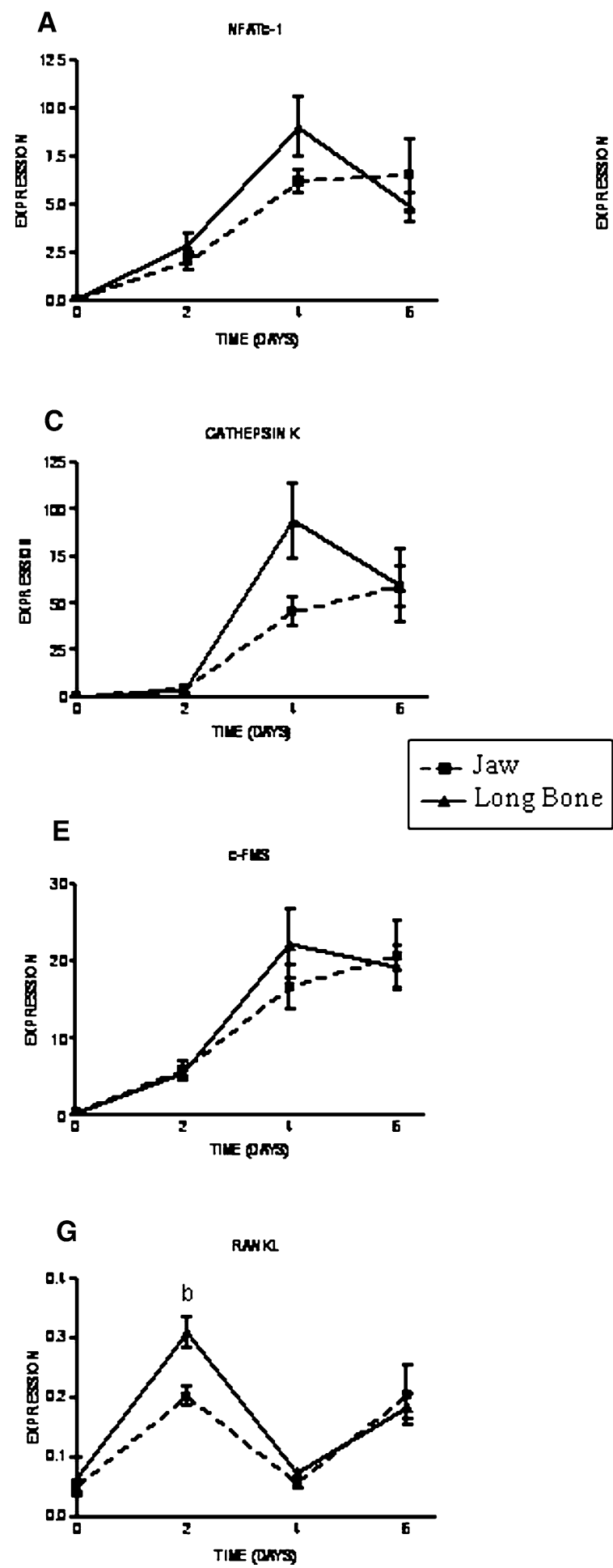

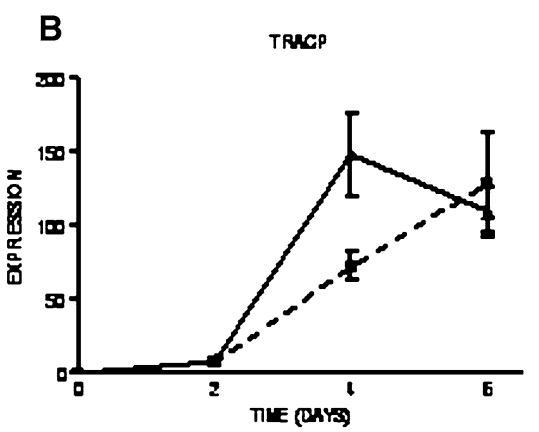

D
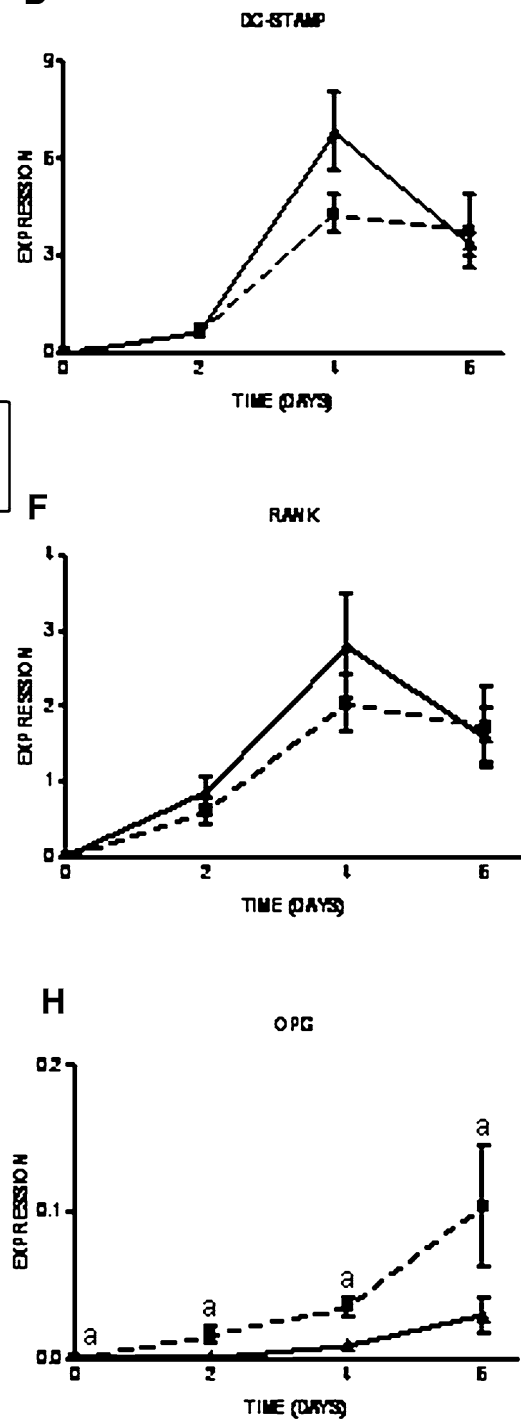

highest $(P<0.01)$ at an early time point ( 2 days) in cultures of long bones (Fig. 4g), whereas OPG gradually increased throughout the culture period (Fig. 4h). OPG expression was significantly higher $(P<0.05)$ in the jaw cultures from day 2 (Fig. $4 \mathrm{~h}$ ). The ratio of RANKL:OPG expression was higher $(P<0.05)$ for the jaw on $t=0$ (Fig. 5a). At days 2 (Fig. 5b) and 4 (Fig. 5c), this ratio was significantly higher $(P<0.01)$ in long bone cultures. On day 6 , there was no significant difference in the RANKL:OPG ratio (Fig. 5d). 
Fig. 5 Ratio of RANKL:OPG expression in jaw and long bone cultures. RANKL:OPG ratio was higher for the jaw at $t=0$ (a), whereas it was significantly higher for long bone cells at 2 (b) and 4 (c) days. On day 6 (d), there was no significant difference between jaw and long bone. Data are expressed as mean $\pm \mathrm{SD} .{ }^{\mathrm{a}} P<0.05$, ${ }^{\mathrm{b}} P<0.01$

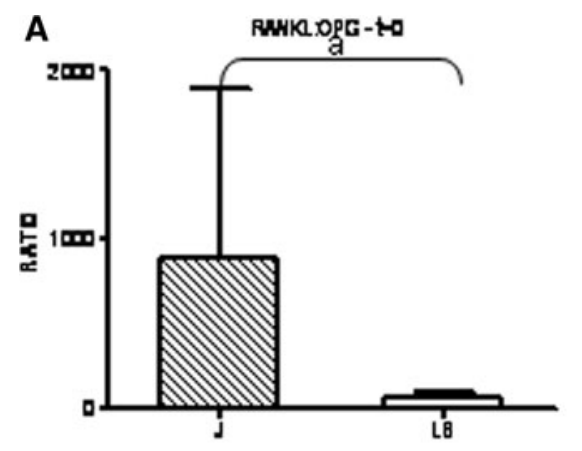

B

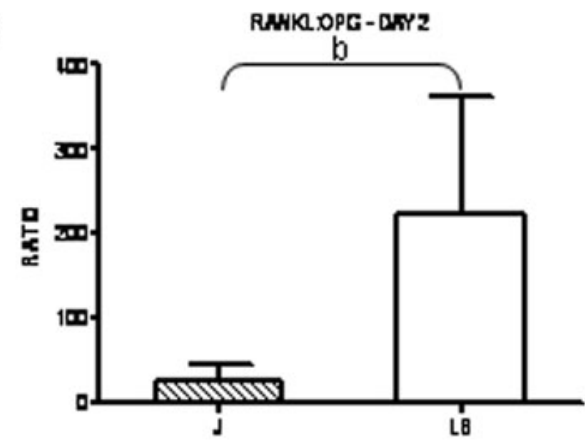

C

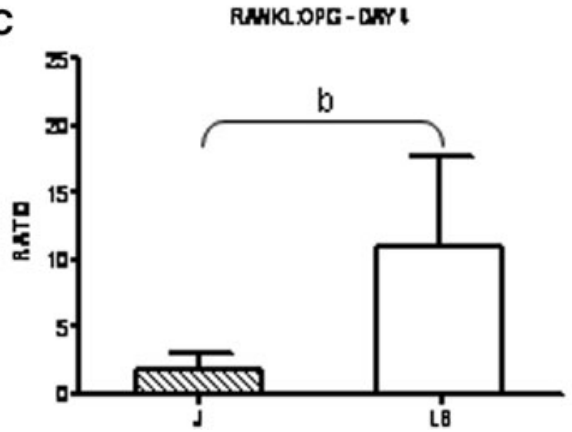

D

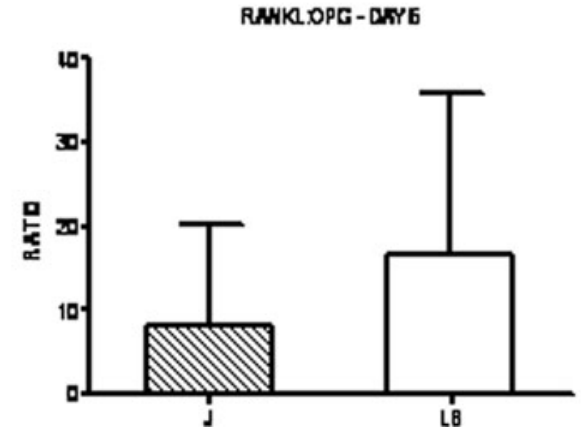

Differences Between TRAP ${ }^{+}$Cells Formed from Jaw and Long Bone Marrow when Seeded on Plastic or Dentin

The data described above were obtained from cells seeded on plastic. We next investigated the osteoclastogenic potential of long bone and jaw precursors on dentin. When the osteoclast precursors were cultured on dentin slices (Fig. 6a, b) for 6 days, the total number of multinucleated osteoclasts formed was similar for jaw and long bone cultures (Fig. 6c). However, the distribution of large (more than 10 nuclei) and small osteoclasts was not similar. The cultures of jaw bone marrow exhibited a higher $(P<0.05)$ number of such large osteoclasts (Fig. 6d). This finding coincides with the culture on plastic, but on dentin the $\mathrm{TRAP}^{+}$multinucleated cells were much larger (at least twofold larger) than those on plastic. Besides the high number of cells with more than 10 nuclei, occasionally $\mathrm{TRAP}^{+}$cells with more than 30 nuclei (Fig. 6a, c) were observed on dentin; this type of cell was not found on plastic.

Although on dentin long bone osteoclasts were smaller than jaw osteoclasts, the former osteoclasts appeared to express a higher TRAP activity (Fig. 6b). The activity of the TRAP enzyme assayed in the supernatants of both cultures confirmed this finding, showing that, from day 3 to day 6 , the long bone osteoclasts released significantly higher $(P<0.05)$ levels of TRAP than the osteoclasts formed from the jaw precursors (Fig. 6f). This difference in
TRAP activity in medium was found only when the cells were seeded on dentin.

The number of osteoclasts generated from jaw precursors was higher $(P<0.05)$ on dentin than on plastic (Fig. 6e); such a difference was not found with long bone cells.

The Different Osteoclasts Resorb Dentin Similarly

When the osteoclast precursors were cultured on dentin slices for 10 days, we found that the resorptive activity of osteoclasts derived from the jaw (Fig. $6 \mathrm{~g}$ ) was higher than that of the long bone osteoclasts (Fig. 6h), but this difference was not statistically significant (Fig. 6i).

\section{Discussion}

The present study provides evidence that the formation of osteoclasts from marrow precursors of jaw bone differs from the formation from long bone marrow precursors. Jaw and long bone precursors differed with respect to the dynamics of the osteoclastogenesis; in the long bone cultures osteoclasts were formed faster than in the jaw cultures. Moreover, jaw osteoclasts proved to be larger and the level of TRAP activity was higher in long bone cells.

The faster generation of long bone osteoclasts was apparent at the 4-day time point; a significantly higher number of $\mathrm{TRAP}^{+}$multinucleated cells were formed in 
Fig. 6 Osteoclasts formed from jaw (a) and long bone

(b) precursors cultured on dentin for 6 days. a A huge osteoclast $(O c)$ that contains more than 50 nuclei.

b Relatively small osteoclast with high cytoplasmic TRAP activity. Bars $=100 \mu \mathrm{m}$.

c Total numbers of multinucleated osteoclasts formed on dentin from jaw and long bone marrow precursors were not different if grouped in the categories as indicated. d However, if all cells with more than 10 nuclei were grouped together, a significantly higher number was found in jaw cultures. e Comparing the cells formed on plastic and dentin, a higher number of jaw osteoclasts were formed on dentin compared to the number formed on plastic. No significant differences were observed for long bone cultures. f Levels of TRAP enzyme secreted in the medium were not different between jaw and long bone cultures on plastic. On dentin long bone osteoclasts released higher levels of TRAP. $\mathbf{g}, \mathbf{h}$ Examples are shown of resorption pits $(R P$, arrows $)$ generated by jaw (g) and long bone (h) marrow precursorderived osteoclasts, until day 10. Bars $=100 \mu \mathrm{m}$.

i Percentage of resorption. After 10 days' culture, the percent of resorption by jaw- and long bone-derived osteoclasts was not different. Percent of resorption was from two experiments ( $n=3$ mice per experiment) and expressed as mean \pm SD. ${ }^{\mathrm{a}} P<0.05$
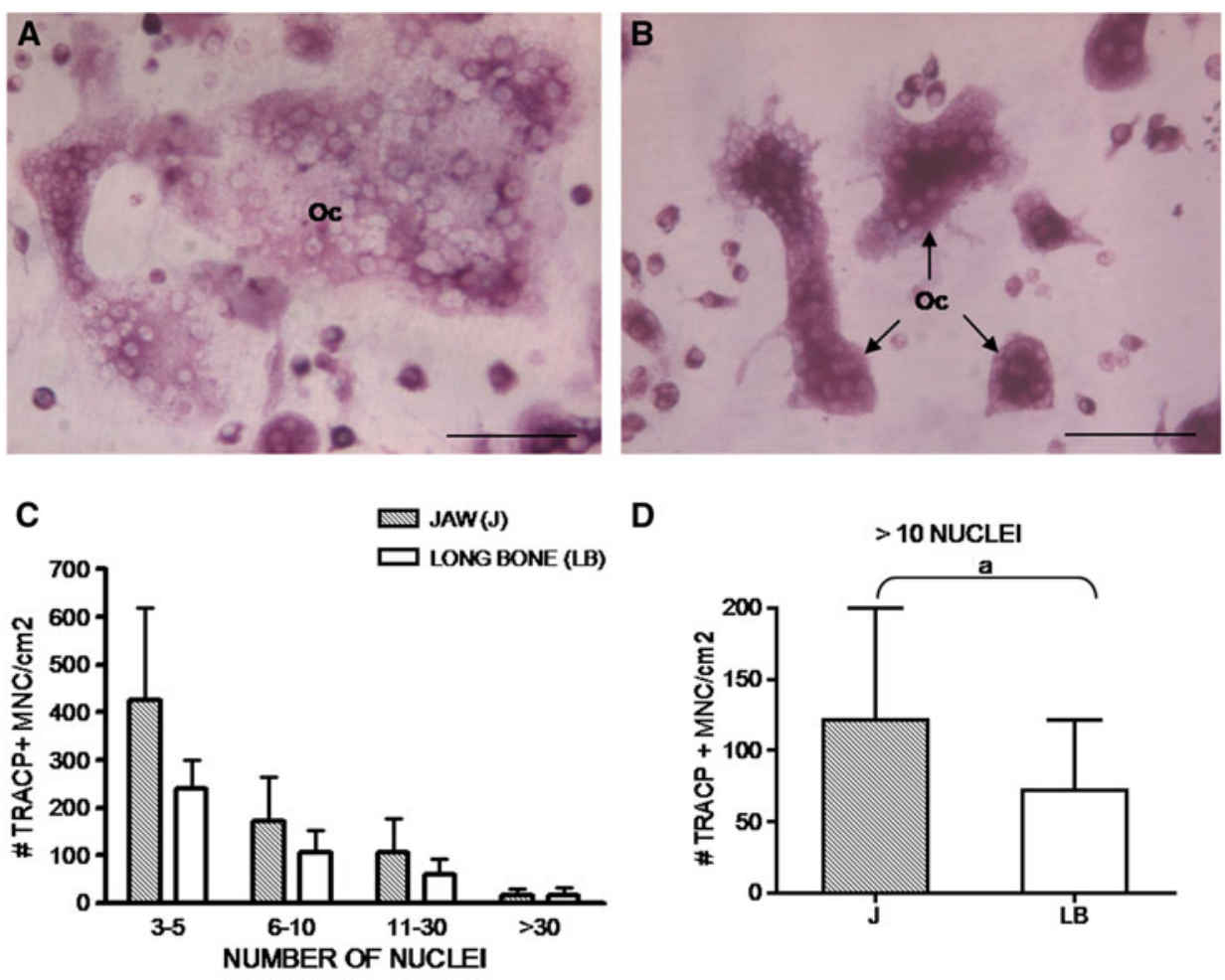

D
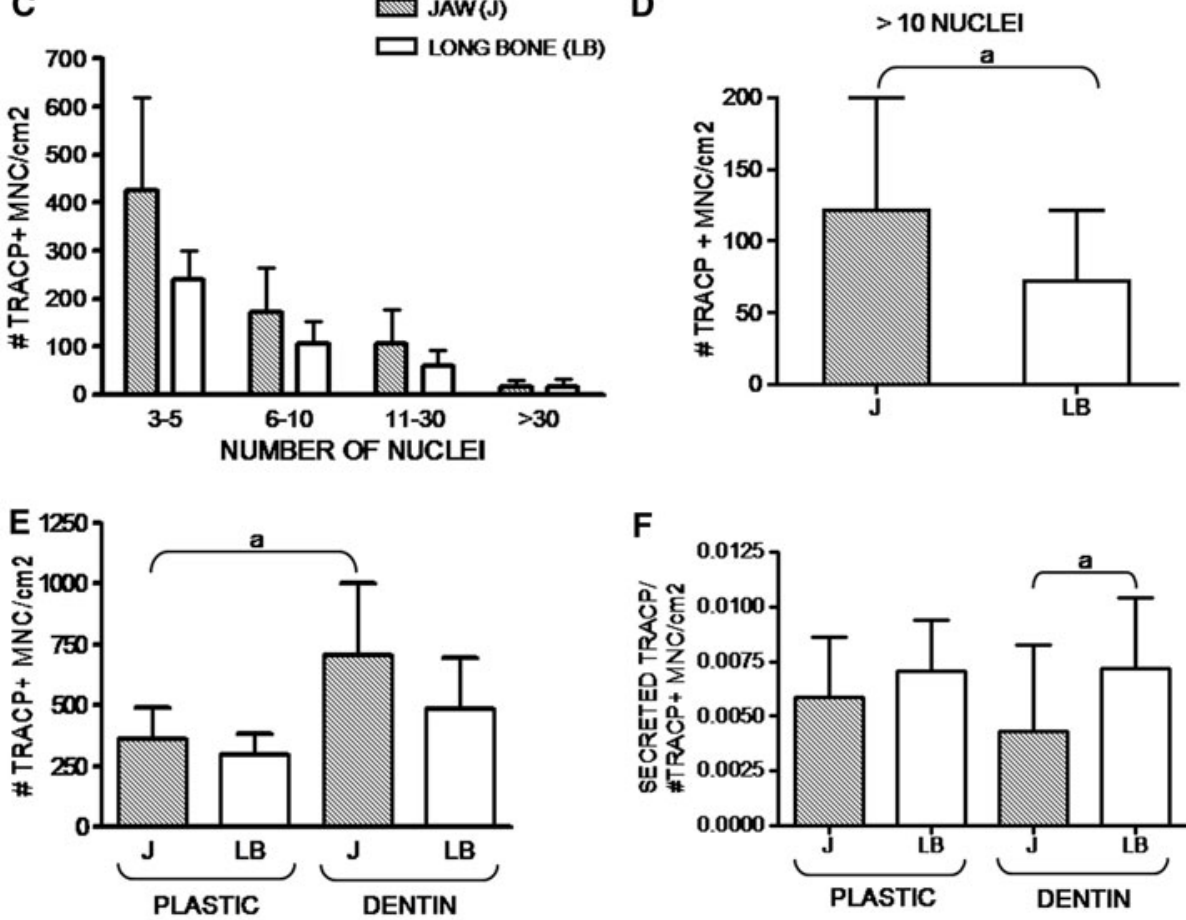

$\mathbf{F}$
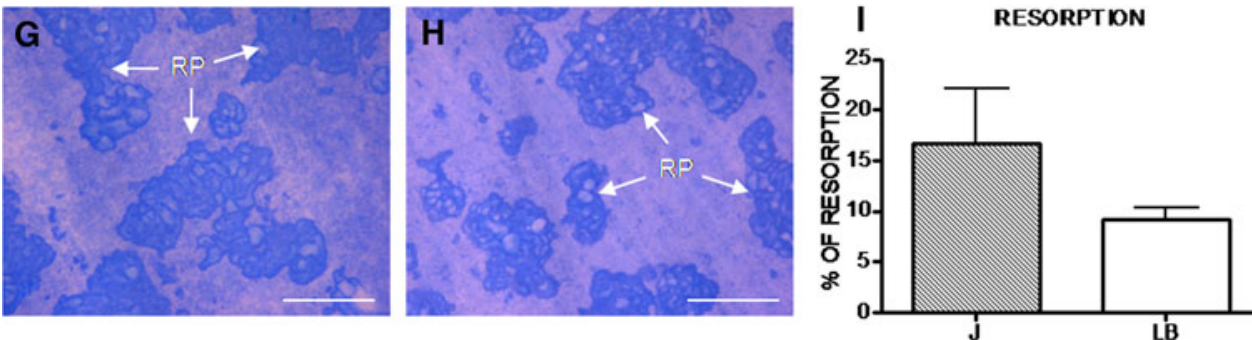

these cultures, whereas in the jaw cultures only a few cells with three or more nuclei were observed. The jaw cultures did, however, catch up. From day 4 to day 6, a threefold increase in the number of TRAP ${ }^{+}$multinucleated cells took place in the jaw cultures and the total number of osteoclastlike cells formed from jaw and long bone precursors was no longer different at the end of the culture period (day 6). However, on day 6, the large cells (with more than 10 nuclei) were more frequently seen in the jaw than in the long bone cultures. This is in contrast to what was observed on day 4 , when almost no large $\mathrm{TRAP}^{+}$cells were found in the jaw cultures. Therefore, our data indicate that the initial formation of osteoclasts from the jaw precursors was slower but they catch up during the onset of culturing and finally form larger osteoclasts.

One of the explanations for the fast osteoclastogenesis and, consequently, early osteoclast formation in the long bone cultures might be the differences in the cellular composition of the two types of bone marrow. The long bone marrow did indeed contain more osteoclast precursor 
cells of the myeloid lineage (early blasts, myeloid blasts, and monocytes) than the jaw bone marrow. In addition, among the myeloid precursors of the long bone marrow, there was a higher number of myeloid blasts than in the jaw bone marrow. It was recently shown that myeloid blasts are the cells that differentiate into osteoclasts in a relatively short period of time [3]. From cultures of this type of precursors, the maximal number of osteoclasts was reached in 4 days, which is in agreement with our findings obtained with the bone marrow cultures from long bone. In the jaw bone marrow, the relative frequency of monocytes was higher than in long bone marrow. In comparison with the myeloid blasts, the monocytes need more time to differentiate into osteoclasts [3].

The kinetic differences in osteoclast-forming capacity between jaw and long bone were also confirmed by analysis of the expression of osteoclast- and osteoclastogenesis-related genes. qPCR analyses revealed different expression patterns for jaw and long bone. Taking into consideration these findings, we propose that the percentage of myeloid precursors in the different bone marrows and the differences in proliferation among the myeloid stages account for the observed differences in the time needed for osteoclast formation in the jaw compared to this process in long bone cultures.

An alternative explanation could be the difference found in expression of RANKL and OPG, proteins that either stimulate or inhibit osteoclastogenesis, respectively [19]. However, since we added an excess of RANKL to our cultures, it is not very likely that the differences found in the genesis of the osteoclasts were due to differences in expression of this molecule.

Not only on plastic but also on dentin, the formation of osteoclasts by the two populations of precursors proved to be different. On dentin, large osteoclasts (with more than 10 nuclei) were more frequently found in the jaw cultures, thus coinciding with the observation on plastic. However, on dentin, the $\mathrm{TRAP}^{+}$cells from jaw cultures were much larger and occasionally contained more than 30 nuclei. In addition, when we compared the effect of the substrate on the occurrence of $\mathrm{TRAP}^{+}$cells generated from the same precursors, we found more jaw osteoclasts on dentin than on plastic, whereas the number of osteoclasts raised from long bone marrow was not affected by the substrate. This finding indicates that the mineralized substrate may have a stronger effect on the osteoclastogenesis from jaw bone marrow and, obviously, on the size of the jaw osteoclasts. A difference in the size of osteoclasts was also noted in bone biopsies from calvaria and long bones obtained from a pyknodysostotic fetus. The calvarial osteoclasts proved to be twice the size of long bone osteoclasts and had approximately two times more nuclei [9].

Analysis of TRAP activity showed that when the cells were cultured on plastic the level of this enzyme in the supernatant was not different between the two types of cultures. The findings on plastic seem to confirm the results obtained with the analysis of jaw and long bone TRAP mRNA. Although from day 2 to day 4 there was a higher increase in the expression of TRAP in the long bone in comparison with the jaw cultures, from day 4 to day 6 there was a decrease in expression in the long bone cultures. Distinctly, the expression in jaw bone marrow cultures increased throughout the culture period, and on day 6 it tended to be higher than in long bone cultures. Therefore, even though the expression patterns of TRAP mRNA by jaw and long bone cells were different, at the end of the culture period the total levels of gene expression were similar.

A remarkable difference in TRAP secretion was observed when the precursors were seeded on dentin. In the long bone cultures on dentin, TRAP activity proved to be higher compared to that in the jaw cultures. The increased secretion of TRAP by long bone cultures strongly suggests functional differences between osteoclasts from different bone sites in terms of participation of the enzymes in the degradation of mineralized tissues. Recently, Zenger et al. [25] showed functional differences between bone-resorbing osteoclasts from different bone sites with respect to the activation of TRAP by cathepsin K. The contribution of TRAP to osteoclast functional heterogeneity was also shown in a study comparing calvaria and long bone [8]. In the latter study, osteoclasts from calvaria proved to release higher levels of TRAP than the ones from long bone. Even though calvaria and jaw are both skull bones, their osteoclasts may be functionally different. It could be that long bone cells secreted more TRAP than jaw cells because the jaw cells are somewhat behind in differentiation. However, the fact that there was no difference between jaw and long bone TRAP secretion on plastic suggests an influence of the substrate on TRAP secretion.

Concerning the bone-resorptive activity, we found a somewhat higher resorption by jaw osteoclasts; but this did not prove to be significant. Studies involving rabbit long bone osteoclasts have shown that osteoclast size is associated with increased resorptive activity or efficiency [26, 27]. Large osteoclasts proved to resorb 2.5 times more per cell than small osteoclasts, but the amount resorbed per nucleus was the same for the two categories [27]. In a study investigating rat long bone osteoclasts, the small osteoclasts exhibited a lower capacity for degradation of bone matrix and a lower expression of MMP-9 and cathepsin K compared to the large osteoclasts [28]. In our study the percentage of large osteoclasts was higher in the jaw cultures but still relatively low. This low percentage may explain why we did find a difference in resorption, but this difference proved not to be significant. Further studies are needed to analyze the bone-resorbing capacity of the 
osteoclasts generated from the different bone marrows in more detail.

A possible explanation for the differences observed between jaw and long bone osteoclasts could be the skeletal site-specific osteogenic properties of the bone marrow cells in orofacial bones (maxilla and mandible) compared to other bones based on their different embryological origins. The orofacial bones are formed by ectomesenchymal cells (originated from the association of neural crest cells and mesoderm) while other bones develop from mesoderm [29]. The distinct origin may, in part, explain the differences between jaw and long bone with respect to the formation and activity of osteoclasts observed in our study. Therefore, the results obtained in the present study provide strong support for the view of osteoclast-bone site heterogeneity, showing that bone marrows from different skeletal locations (jaw and long bone) result in different dynamics of osteoclastogenesis and possible functional differences between osteoclasts.

Acknowledgments The authors thank CAPES (BEX:1174/08-8, Brazil) and CNPq (Brazil) for financial support. Dr. Lars Mulder (Functional Anatomy, Academic Center for Dentistry Amsterdam, Amsterdam, The Netherlands) is acknowledged for providing the microCT illustration of Fig. 1.

Open Access This article is distributed under the terms of the Creative Commons Attribution Noncommercial License which permits any noncommercial use, distribution, and reproduction in any medium, provided the original author(s) and source are credited.

\section{References}

1. Quinn JMW, Neale S, McGee JOD, Athanasou NA (1998) Human osteoclast formation from blood monocytes, peritoneal macrophages, and bone marrow cells. Calcif Tissue Int 62: 527-531

2. Jacquin C, Gran DE, Lee SK, Lorenzo JA, Aguila HL (2006) Identification of multiple osteoclast precursor populations in murine bone marrow. J Bone Miner Res 21:67-77

3. De Vries TJ, Schoenmaker T, Hooibrink B, Leenen PJ, Everts V (2009) Myeloid blasts are the mouse bone marrow cells prone to differentiate into osteoclasts. J Leukoc Biol 85:919-927

4. Everts V, de Vries TJ, Helfrich MH (2009) Osteoclast heterogeneity: lessons from osteopetrosis and inflammatory conditions. Biochim Biophys Acta 1792:757-765

5. Everts V, Korper W, Jansen DC, Steinfort J, Lammerse I, Heera S, Docherty AJ, Beertsen W (1999) Functional heterogeneity of osteoclasts: matrix metalloproteinases participate in osteoclastic resorption of calvarial bone but not in resorption of long bone. FASEB J 13:1219-1230

6. Shorey S, Heersche JN, Manolson MF (2004) The relative contribution of cysteine proteinases and matrix metalloproteinases to the resorption process in osteoclasts derived from long bone and scapula. Bone 35:909-917

7. Everts V, Korper W, Hoeben KA, Jansen ID, Bromme D, Cleutjens KB, Heeneman S, Peters C, Reinheckel T, Saftig P, Beertsen W (2006) Osteoclastic bone degradation and the role of different cysteine proteinases and matrix metalloproteinases: differences between calvaria and long bone. J Bone Miner Res 21:1399-1408

8. Perez-Amodio S, Jansen DC, Schoenmaker T, Vogels IM, Reinheckel T, Hayman AR, Cox TM, Saftig P, Beertsen W, Everts V (2006) Calvarial osteoclasts express a higher level of tartrate-resistant acid phosphatase than long bone osteoclasts and activation does not depend on cathepsin $\mathrm{K}$ or $\mathrm{L}$ activity. Calcif Tissue Int 79:245-254

9. Everts V, Hou WS, Rialland X, Tigchelaar W, Saftig P, Bromme D, Gelb BD, Beertsen W (2003) Cathepsin K deficiency in pycnodysostosis results in accumulation of non-digested phagocytosed collagen in fibroblasts. Calcif Tissue Int 73:380-386

10. Zaidi M, Moonga B, Moss DW, MacIntyre I (1989) Inhibition of osteoclastic acid phosphatase abolishes bone resorption. Biochem Biophys Res Commun 159:68-71

11. Moonga BS, Moss DW, Patchell A, Zaidi M (1990) Intracellular regulation of enzyme secretion from rat osteoclasts and evidence for a functional role in bone resorption. J Physiol 429:29-45

12. Hayman AR, Jones SJ, Boyde A, Foster D, Colledge WH, Carlton MB, Evans MJ, Cox TM (1996) Mice lacking tartrate-resistant acid phosphatase (Acp 5) have disrupted endochondral ossification and mild osteopetrosis. Development 122:3151-3162

13. Suter A, Everts V, Boyde A, Jones SJ, Lüllmann-Rauch R, Hartmann D, Hayman AR, Cox TM, Evans MJ, Meister T, von Figura K, Saftig P (2001) Overlapping functions of lysosomal acid phosphatase (LAP) and tartrate-resistant acid phosphatase (Acp5) revealed by doubly deficient mice. Development 128:4899-4910

14. Roberts HC, Knott L, Avery NC, Cox TM, Evans MJ, Hayman AR (2007) Altered collagen in tartrate-resistant acid phosphatase (TRAP)-deficient mice: a role for TRAP in bone collagen metabolism. Calcif Tissue Int 80:400-410

15. Hollberg K, Hultenby K, Hayman A, Cox T, Andersson G (2002) Osteoclasts from mice deficient in tartrate-resistant acid phosphatase have altered ruffled borders and disturbed intracellular vesicular transport. Exp Cell Res 279:227-238

16. Jansen ID, Mardones P, Lecanda F, de Vries TJ, Recalde S, Hoeben KA, Schoenmaker T, Ravesloot JH, van Borren MM, van Eijden TM, Bronckers AL, Kellokumpu S, Medina JF, Everts V, Oude Elferink RP (2009) Ae2(a,b)-deficient mice exhibit osteopetrosis of long bones but not of calvaria. FASEB J 23: 3470-3481

17. Van den Bos T, Speijer D, Bank RA, Brömme D, Everts V (2008) Differences in matrix composition between calvaria and long bone in mice suggest differences in biomechanical properties and resorption: special emphasis on collagen. Bone 43:459-468

18. Sodek KL, Tupy JH, Sodek J, Grynpas MD (2000) Relationships between bone protein and mineral in developing porcine long bone and calvaria. Bone 26:189-198

19. Kobayashi Y, Udagawa N, Takahashi N (2009) Action of RANKL and OPG for osteoclastogenesis. Crit Rev Eukaryot Gene Expr 19:61-72

20. Chung H, Kang YS, Hwang CS, Moon IK, Yim CH, Choi KH, Han KO, Jang HC, Yoon HK, Han IK (2001) Deflazacort increases osteoclast formation in mouse bone marrow culture and the ratio of RANKL/OPG mRNA expression in marrow stromal cells. J Korean Med Sci 16:769-773

21. Odgren PR, Kim N, MacKay CA, Mason-Savas A, Choi Y, Marks SC Jr (2003) The role of RANKL (TRANCE/TNFSF11), a tumor necrosis factor family member, in skeletal development: effects of gene knockout and transgenic rescue. Connect Tissue Res 44:264-271

22. Hoff AO, Toth BB, Altundag K, Johnson MM, Warneke CL, Hu M, Nooka A, Sayegh G, Guarneri V, Desrouleaux K, Cui J, Adamus A, Gagel RF, Hortobagyi GN (2008) Frequency and risk 
factors associated with osteonecrosis of the jaw in cancer patients treated with intravenous bisphosphonates. J Bone Miner Res 23:826-836

23. Stefanik D, Sarin J, Lam T, Levin L, Leboy PS, Akintoye SO (2008) Disparate osteogenic response of mandible and iliac crest bone marrow stromal cells to pamidronate. Oral Dis 14:465-471

24. De Bruijn MFTR, Slieker WAT, van der Loo JCM, Voerman JSA, Van Ewijk W, Leenen PJM (1994) Distinct mouse bone marrow macrophage precursors identified by differential expression of ER-MP12 and ER-MP20 antigens. Eur J Immunol 24:2279-2284

25. Zenger S, Hollberg K, Ljusberg J, Norgård M, Ek-Rylander B, Kiviranta R, Andersson G (2007) Proteolytic processing and polarized secretion of tartrate-resistant acid phosphatase is altered in a subpopulation of metaphyseal osteoclasts in cathepsin $\mathrm{K}$ deficient mice. Bone 41:820-832
26. Lees RL, Heersche JN (2000) Differences in regulation of $\mathrm{pH}(\mathrm{i})$ in large ( $\geq 10$ nuclei) and small ( $\leq 5$ nuclei) osteoclasts. Am J Physiol Cell Physiol 279:C751-C761

27. Lees RL, Sabharwal VK, Heersche JN (2001) Resorptive state and cell size influence intracellular $\mathrm{pH}$ regulation in rabbit osteoclasts cultured on collagen-hydroxyapatite films. Bone 28:187-194

28. Hu Y, Ek-Rylander B, Karlström E, Wendel M, Andersson G (2008) Osteoclast size heterogeneity in rat long bones is associated with differences in adhesive ligand specificity. Exp Cell Res 314:638-650

29. Akintoye SO, Lam T, Shi S, Brahim J, Collins MT, Robey PG (2006) Skeletal site-specific characterization of orofacial and iliac crest human bone marrow stromal cells in same individuals. Bone 38:758-768 